\title{
ÜBER DIE BEGATTUNG UND UBBER DEN STYLUS DER AGROMYZIDEN
}

\author{
VON \\ J. C. H. DE MEIJERE \\ (Amsterdam)
}

Als Festschrift zur Feier der Königl. Zoolog. Gesellschaft Natura Artis Magistra kann die Wahl des Themas: die Begattung einer ca. $2 \mathrm{~mm}$. grossen Fliege, befremden. Zwei Umstände mögen diese rechtfertigen. Zunächst, dass Kopulationen in der Gieschichte des Zoologischen Gartens so ausserordentlich wichtig gewesen sind. Schon seit langer Zeit gelang wiederholt die Fortpflanzung des Nilpferdes und in den späteren Jahren ist die Kopulation bei immer mehr Tieren durch guten Erfolg gekrönt worden, namentlich auch bei den grösseren Raubtieren und in den letzten Jahren beim Wisent. Andererseits handelt es sich um eine kleine Fliege die im Garten, wie auch sonst in Holland, besonders häufig auftritt, die Minierfliege der Stechpalme (Ilex), die seit vielen Jahren durch ihre im Winter in den Blättern lebenden Larven mir schönes frisches Material für meine Praktika im Zoolog. Laboratorium der Amsterdamer Universität lieferte. Es ist mir eine Freude, über einen so besonderen Lebensakt dieser Fliege mit den zahlreichen, dabei eine Rolle spielenden Chitingebilden an dieser Stelle etwas mitteilen zu können.

Wegen der eigentümlichen Bildung der letzten Hinterleibssegmente mit ihrem Rasp-Apparat bei den Weibchen der Agromyziden erschien es erwünscht die Kopulation dieser Fliegen eingehender $z u$ studieren. Im allgemeinen trifft man diese nicht oft in diesem Zustande und bei Beunruhigung lösen sie sich meistens bald. Mein Schüler, Herr E. A. M. Speijer hat in seiner InauguralDissertation: „De hypopygia van eenige Agromyzidae, benevens theoretische beschouwingen over de homologieën van de aanhangsels hiervan (1934)", das Verhalten dieser Chitingebilde an einer Reihe von Phytomyza-Arten untersucht. Die Kopulation wahrzunehmen gelang ihm aber nicht. Ich beobachtete diese bei Dizygomyza abnormalis Mall. im Freien Mitte Juni einige Male, meistens zwischen 12-4 Uhr bei sonnigem Wetter im Garten des UniversitätsInsectarium zu Amsterdam an einer mit Chenopodium album bewachsenen Stelle, deren Stengel mehrere Jahre hindurch von dieser Art stark befallen waren. Dann gelang es mir auch im der zweiten Hälfte von Mai eingefangene Phytomyza ilicis Curt., die an Ilex aquifolium hier sehr allgemein ist und 
platzartige Minen verursacht, einige Tage mit Zuckerkörnern in einer weiten Tube lebend $z u$ behalten und auch einige Male die Begattung wahrzunehmen. Solche Pärchen von Phytomyza ilicis nahm ich in der Glastube meistens ca. 3 Uhr im Nachmittag wahr, als die Sonne schien. Während der Kopulation sitzt das $\sigma^{\prime}$ auf dem $Q$, mit seinen Vorderbeinen auf dem Kopf oder Thorax des $ᄋ$, dessen Flügel etwas ausgespreizt sind. Diese Pärchen, sowie auch die eingefangenen von Diz. abnormalis gelang es mir auch, obgleich nicht immer, vermittels eines in Chloroform getauchten Pinsels in diesem Zustande schnell zu töten, ohne das Lösung eintrat. Nach Fixierung werden die abgeschnittenen, aber noch zusammenhängenden :Hinterleibsenden in Phenolum liquidum zur mikroskopischen Untersuchung genügend durchsichtig.

Zunächst möge hier über den verwickelten Bau des Hinterleibsendes dieser winzigen, meistens nur $1,5-2 \mathrm{~mm}$ langen Fliegen im allgemeinen etwas gesagt sein. Die Figuren I (von Dizygomyza abnormalis Mall.) und 2 (von Phytomyza ilicis Curt.) können hierbeì als Schema dienen.

Beim $Q$ ist im Ruhezustande der 7 te ringsum chitinisierte Ring der letzte sichtbare und bildet die Legeröhre, der 8te und gte können aus ihm hervorgebracht werden. Dem 7ten voran' gehen je 6 Tergite und 6 Sternite. Das 8te Segment trägt das komplizierte Rasp-Apparat, das wohl nach den Arten verschieden ist und nach meinem Befund bei im Stengel minierenden Melanagromyza-Arten auch säge-artige Gebilde enthalten kann; am Ende dieses Segments finden sich die Genitalöffnung und 2 blattförmige Gebilde, die valvulae inferiores; das 9te Segment ist sehr schmal und parallelrandig, fast ganz nackt; es trägt am hinteren Ende die Analöf fnung und die beiden eingliedrigen cerci, weil ein gesondertes Iotes Segment nicht zu erkennen ist.

Die dünne Vagina durchläuft das ganze 7te und 8te Segment. Am Anfang des 7ten nimmt sie die gemeinsame Endpartie der Ausführgänge der beiden flach halbkugelförmigen, gewöhnlich hier sehr dunkel gefärbten receptacula seminis sowie die Ausführgänge zweier grossen Drüsen auf, während nach Miall und TAYLOR ventral noch ein kleines Säckchen mit kurzem Gang mit ihr verbunden ist. Eigentümlich ist noch, dass das 7 te Tergit sich nach vorne hin als eine trapezförmige Platte mit nach unten umgebogenen Seitenrand unter dem Tergit des 6ten fortsetzt.

Aus der Lage der weiblichen Genitalöffnung folgt schon, dass das 9te Segment nicht an die Kopulation teilnimmt. Beim kopulierten Pärchen ist es vollständig ausgetreten und liegt lose neben dem Hinterleibsende des $\sigma^{\circ}$. Ferner ergibt sich, dass der ganze Penis in die Vagina des $q$ gebracht wird und sogar noch mehr als dieser allein; selbst der unten genannte "Chitinstab" ist zum Teil in sie eingebracht, natürlich durch die elastische Haut der betreffenden Stelle des $\delta$ umgeben, denn er liegt ganz im Inneren des Körpers. Namentlich in der Figur von Dizygomyza abnormalis ist zu sehen wie der Yenıs ganz in den 7 ten Hinterleibsring des $Q$ vorgedrungen ist und die distale Hälfte des Chitinstabs sich fast bis zum Vorderende des 8 ten Ringes erstreckt. In der Figur von $P h$. 
ilicis liegt der Penis noch ganz im 8ten Segment, aber der Stab ist noch gar nicht ausgestochen; vielleicht hatten sich die Tiere wegen des Chloroformduftes schon etwas gelöst.

Beim $\sigma$ gehen an den Genitalringen 6 Tergite vorab, von welchen die ersten zwei verschmolzen sind ; vom Genitalapparat ist äusserlich hauptsächlich sichtbar der 9te Ring (nach Schräder's Zählung; der 8te nach Brüel und PeTzOLD), er trägt den in dieser Familie äusserst komplizierten Penis, innen

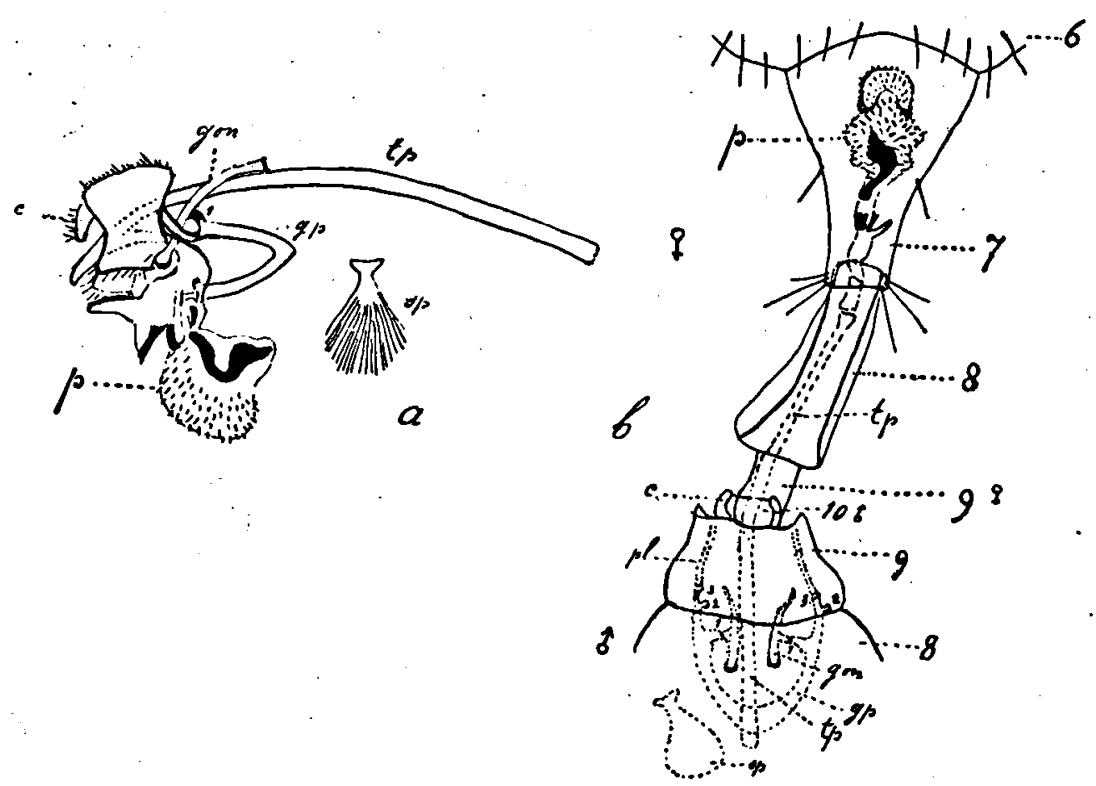

Fig. I. Dizygomyza abnormalis Mall. a. Genitalien am Hinterleibsende des $\delta$, b. $f$ und $q$ in Kopulation; in beiden Abbildungen: 7,8 , 9, 10: die letzten Hinterleibssegmente, $p$ : penis, $c$ : cerci, $t p$ : Tragplatte, $g p$ : Gabelplatte, $1,2,3$ : die 3 Gelenke der Gabelplatte, $s p$ : Samenspritze, gon: Gonapophyse, $p l$ : processus longus..

findet sich im Hinterleibsende dessen Gestell und weitere morphologisch schwer deutbare Chitinstücke, von welchen hier ein langer Stab (die Tragplatte), ein hufeisenförmiges Stück (die Gabelplatte), und ein schulterblattähnliches Gebilde (die Samenspritze) besonders auffallen. Die morphologische Bedeutung dieser Gebilde ist noch nicht ganz sicher. Die ventrale Wand ist nach dem 6ten Sternit fast ganz dünnwandig und eigentliche Sternite sind nicht zu erkennen. Namentlich Brüel, Feuerborn, Schräder und Frl. Petzold haben sich bei Calliphora, bzw. den Tachiniden, bemüht ihre Reste in den Apodemen, nach innen gerichteten Chitinstücken, zu finden. So nimmt SchräDER an, dass das Sternit des 7ten Ringes durch die Gabelplatte repräsentiert wird, das des 8ten durch die Tragplatte, das des gten durch die Samenspritze, und darauf folgt dann 
das sehr kurze Iote Segment, welches den Anus und die cerci trägt. (Bei BrüeL fehlt das 7 te, und er numeriert die folgenden Segmente um eins weniger).
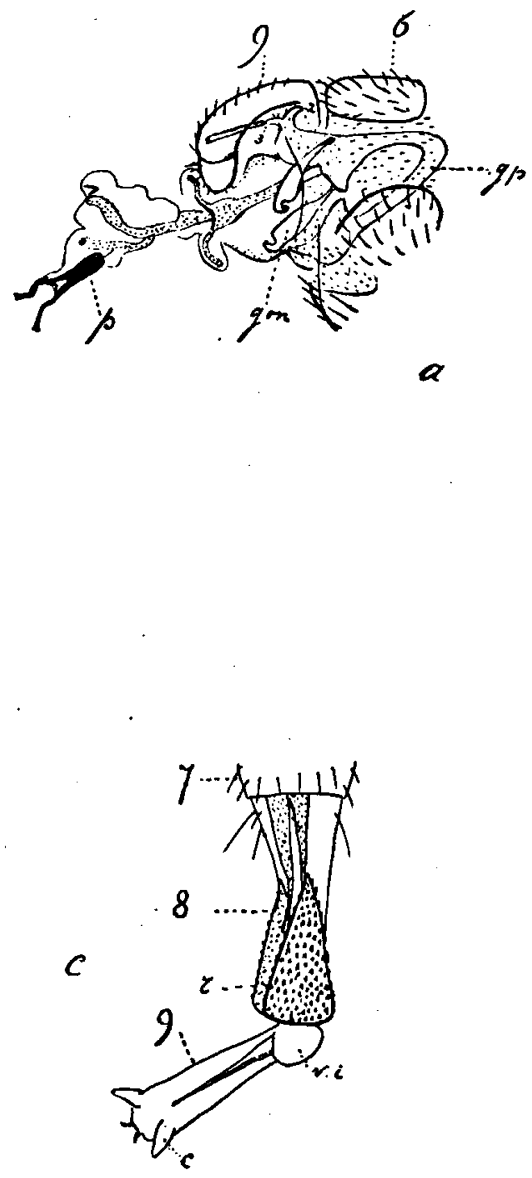
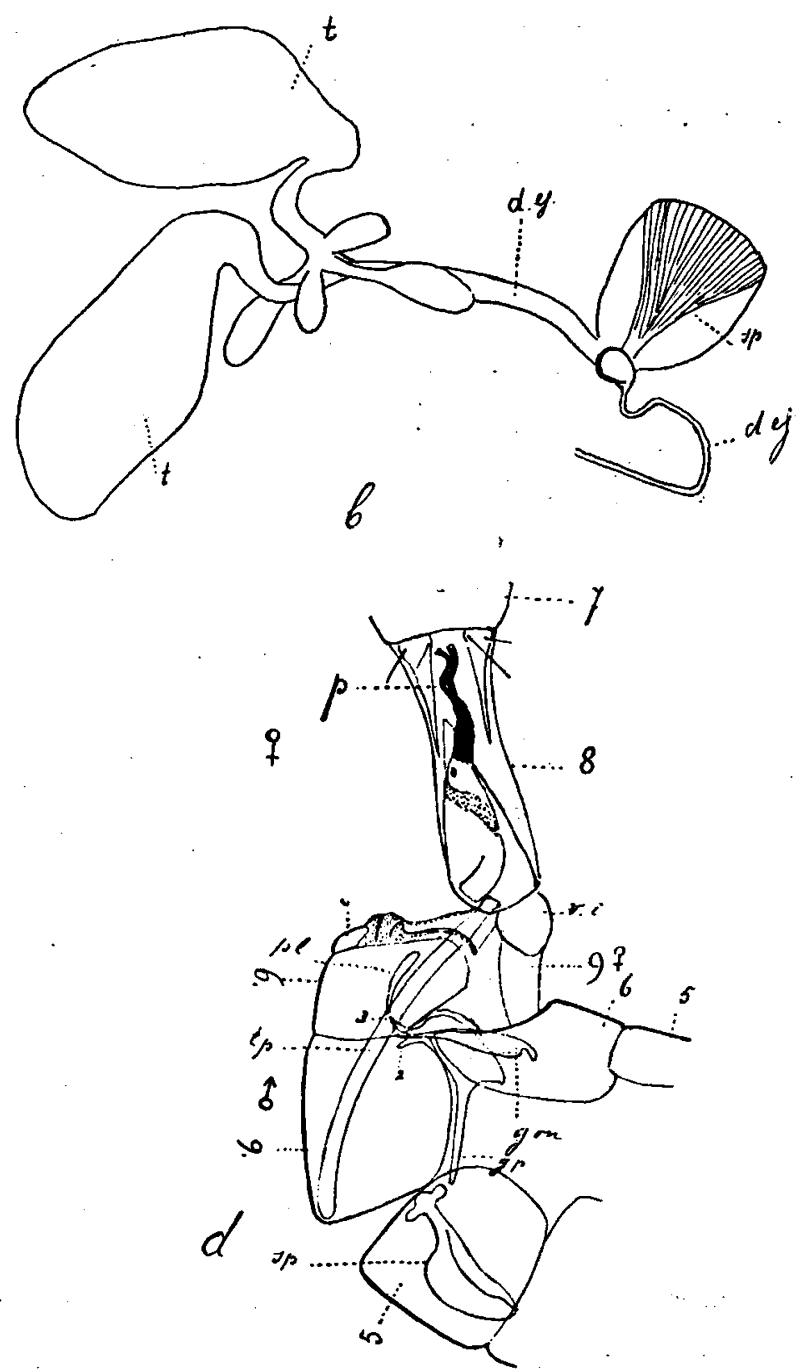

Fig. 2. Phytomyza ilicis Curt. $a$. Hinterleibsende des $\hat{\jmath}, b$. innere Genitalien des $\hat{\delta}$, c. Hinterleibsende des $q, d$. $\delta$ und $q$ in Kopulation. In allen Figuren 5-10: die letzten Hinterleibssegmente, $p$ : penis, $c$ : cerci, $t p$ : Tragplatte, $g p:$ Gabelplatte, I, 2, $3:$ die Gelenke der Gabelplatte, $s p$ : Samenspritze, gon: Gonapophyse, $p l$ : processus longus, $r$ : RaspApparat, vi: valvula inferior, $t$ : testis, $d e$ : ductus ejaculatorius.

Die allmähliche Drehung der Genitalsegmente bei so einem „Hypopygium circumversum" der calyptraten Musciden während der Entwicklung bei der Puppe hat Schräder auf Schnitte verfolgt; da die Drehung endlich $360^{\circ}$ beträgt, liegen die Apodemen dann wieder ventral. 
Eigentümlich für die Agromyziden ist die besondere Länge des Chitinstabes, welcher of fenbar die „Tragplatte” vertritt und bis vorn im 4ten Segment sich erstrecken kann; auch die „Gabelplatte” ist sehr deutlich, hufeisenförmig, weil sic in der Mitte viel weniger breit ist als bei Calliphora. Die stark muskulöse Samenspritze wird nach einer kurzen Strecke vom ductus ejaculatorius erreicht ; ihre chitinöse Stütze ist das „Schulterblatt" SPEIJER's; weiterhin verläuft dieser Ductus längs den Chitinstab nach hinten und tritt in den Penis ein.

Überdies gehören zum Genitalapparat noch ein oder zwei Paar Gonapophysen und jederseits ein Stylus. Die Nomenclatur dieser Teile scheint mir noch nicht ganz sicher. Ein paar Anhänge neben der Penisbasis findet man gewöhnlich leicht und ich habe diese früher als Styli bezeichnet, sowie auch Speijer es später tat. Es sind aus der Chitinhaut ohne Gelenk hervorragende Gebilde, deren unteres Ende sich noch als Apodem im Körper fortsetzt.

Der Stylus ist aber typisch ein gelenkig mit dem 9ten Segment verbundenes Organ, bei dem Schema von Calliphora wird es von ScHRÄDER u.a. als „valvula lateralis" bezeichnet.

Am besten kann man sich über die Verhältnisse orientieren, wenn man die distalen Enden der Gabelplatte betrachtet. Bei Calliphora (SCHRÄDER, Das Hypopygium „circumversum" von Calliphora erythrocephala p. 14, 16, Fig. 4) zeigen diese 3 Gelenkflächen. Die Iste artikuliert mit dem distalen Ende der Basis der Paramere (ibid. p. 14) welche of fenbar eine Gonapophyse ist, die 2te mit einer hier stark vorspringenden Stelle des Vorderrandes des 9ten Tergits, die $3^{\text {te }}$ mit dem proximalen Ende des processus longulus, eines Stabes, dessen distales Ende mit der Basis der valvula lateralis (= Stylus mehrerer Autoren, auch nach meiner Bezeichnung) in Verbindung tritt. Diese 3 Gelenkflächen findet man bei Agromyzinen auch und diese sind die Stellen, welche ich in den Figuren als I, 2 und 3 angegeben habe. Nur zeigt hier der Vorderrand des gten Tergits keinen Fortsatz, und ist das distale Ende des processus longus etwas verlagert weil hier kein besonders abgetrennter Stylus vorhanden ist; es liegt hier nahe dem Hinterrande des gten Tergits. Hieraus erfolgt, dass was ich früher bei der Liriomyza-pusilla-Gruppe (Die Larven der Agromyzinen I. Tijdschr. v. Ent. 1925. p. 289) und auch SPEIJER in seiner Inaug. Dissertation, 1934, bei Agromyzinen als Stylus bezeichnete, eine Gonapophyse ist, während der Stylus mit dem gten Tergit verschmolzen ist und wohl in dem am meisten seitlich liegenden Abschnitt des gten Tergits zu erblicken ist. Bei mehreren Agromyza-Arten (z. B. A. ambigua) ist dieser Abschnitt durch starke kurze Dornen ausgezeichnet, eine deutliche Grenze ist aber dorsal nicht zu erkennen. Somit würde CoLE recht haben, wenn er in seiner Arbeit über „The terminal abdominal structures of Male Diptera" behauptet, dass bei den Agromyzinen der Stylus fehlt, d. h. als gut abgetrenntes Organ, wie man ihn auch bei vielen Acalyptraten findet, und wie ich ihn auch in meiner Arbeit über die Genitalorgane von Anthomyza gracilis Fall. und sordidella Zett. gefunden habe (Tịdschr. v. Entom. LXXV, 1932, p. 284). Ich will hier noch bemerken, dass 
auch bei anderen Formen der Stylus als besonderes Organ verschwinden kann; so sind nach Sturtevant (North American Species of Drosophila, 1921) auch bei Drosophila-Arten die Verhältnisse verschieden. Nach seiner Figur auf p. 34 ist bei Drosophila Buscki Coq. und melanogaster Mg. der „clasper" welche hier den Stylus darstellt, deutlich abgetrennt, bei Dros. funebris. F. ist er mit dem genital arch (dem gten Tergit) verschmolzen.

Bei Dizygomyza abnormatis ist die proximale Hälfte des. Penis (auch die „Theca" genannt), von einem schmalkonischen Vorsprung versehen, worüber einige schwarze Chitinfleckchen stehen, die blasenförmige Endhälfte ist namentlich hinten dicht mit kleinen schwarzen Börstchen besetzt, vorn zeigt sie zwei geschlängelte, nach aussen verbreiterte schwarze Stellen.
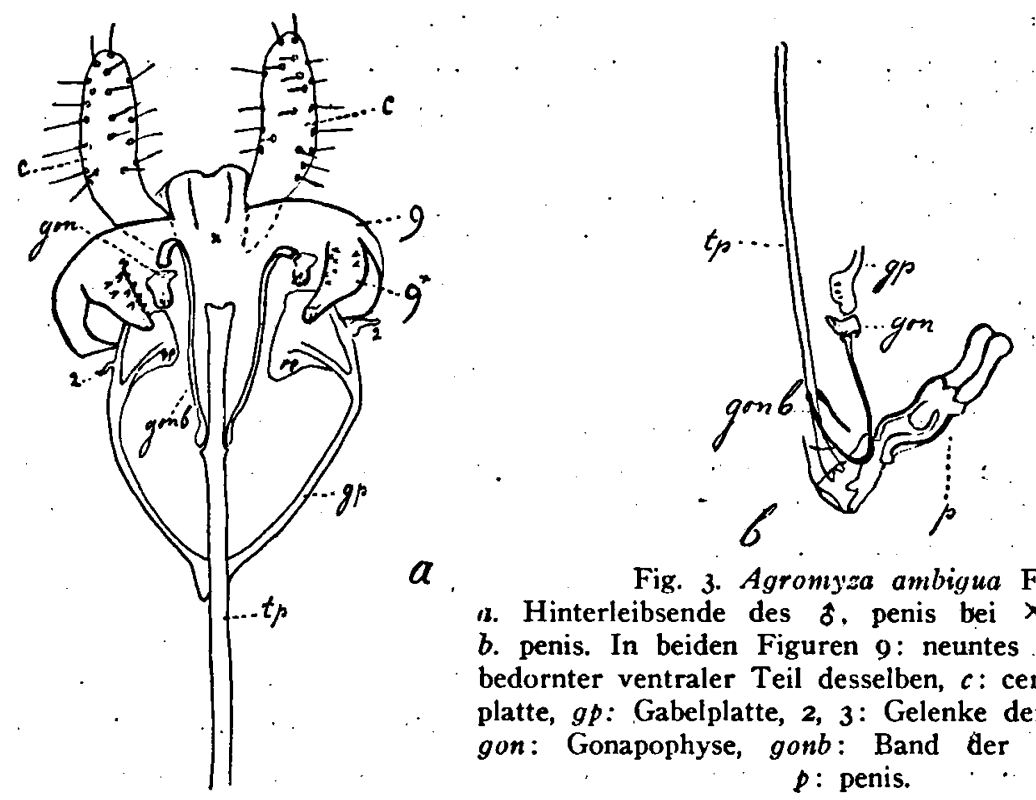

$a$

Fig. 3. Agronyza ambigua Fall.

13. Hinterleibsende des $\delta$, penis bei $x$ abgetrennt, b. penis. In beiden Figuren 9: neuntes Segment, $9^{*}$ : bedornter ventraler Teil desselben, $c$ : cerci, $t p$ : Tragplatte, $g p$ : Gabelplatte, 2, 3: Gelenke der Gabelplatte, gon: Gonapophyse, gonb: Band der Gonapophyse, $p$ : penis.

Der Penis von Phytomyza ilicis hat eine Theca, welche vorne eine dunkle Chitinstrieme zeigt, die am Ende gegabelt ist. Auf die Theca folgt der Körper des Penis, welcher vorn schwarz ist und nach oben ausläuft in 2 schwarze schmale fadenförmige Anhänge, zwischen deren Basis (nach SPEIJER) der ductus ejaculatorius ausmündet. Die hintere Begrenzung des gten Ringes zeigt median eine braune, chitinisierte, ungefähr quadratische Platte, aussen mit 3 kurzen, stumpfen Zähnen und etwas dahinter mit 2 Höckern. Nach SPEIJER p. 6, läge diese Platte eigentlich im Innern und werde durch Zerstörung der sie überdeckenden dünnen Hautschicht nach aussen gepresst; dem kann ich nicht bcistimmen und bin der Ansicht, dass sie von vornherein an der Aussenseite des Körpers liegt. Der Chitinstab endet nicht in ihr, aber gerade unter ihr, ohne verschmolzen zu sein, sodass er' gesondert in die weibliche Genitalöffnung tre- 
ten kann.' Obigen Betrachtungen möchte ich noch einige Bemerkungen über Agromyza hinzufügen.

Schwierige Verhältnisse zeigt die Gattung Agromyza Fall: Von dieser finden sich in Venturi's Abhandlung: „Contributi alla conoscenza dell' Entomofaura delle Graminacee coltivate e spontanee, IV, 1936", die Befunde bei Agromyza (Domomyza) mobilis Mg. beschrieben und durch Figuren erleuchtert (namentlich Fig. 2, 3, 5 und 6, p. I2).

Vergleichend sind die Verhältnisse indessèn von ihm nicht betrachtet, so dass eine Untersuchung in dieser Hinsicht mir nicht überflüssig schien. Die Figuren

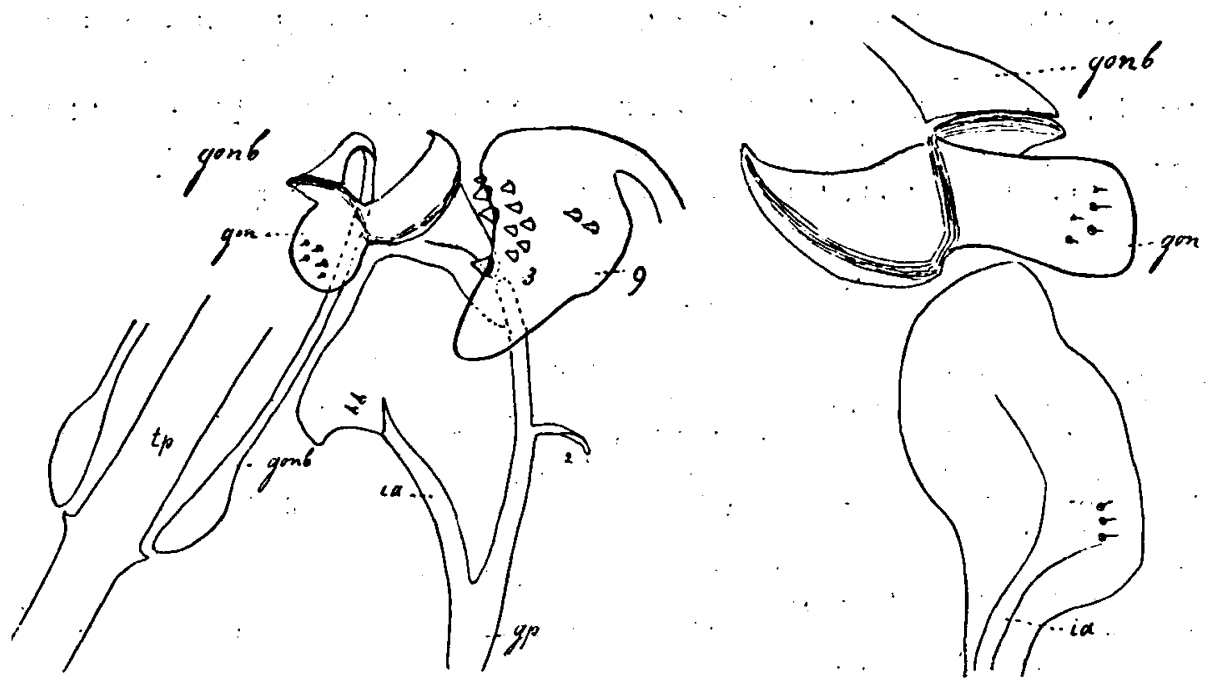

Fig. 4. Agromyza ambigua Fall \&. Teile vom Hinterleibsende, stärker vergrössert; in beiden Figuren 9: ventraler Teil des gten Segments, $t p$ : Tragplatte, $g p$ : Gabelplatte, 2, 3: Gelenke an den Endteilen der Gabelplatte, ia: innerer Ast des distalen Endes der Gabelplatte, gon: Gonapophyse, gonb: Band der Gonapophyse.

3 und 4 beziehen sich auf die sehr ähnliche Agromyza ambigua Fall. Die Gabelplatte ist hier an den distalen Enden breit und trägt ein paar Börstchen, liegt hier also an der Oberfläche der Ventralseite; die 3 Äste, worin sie sich teilt, sind z. T. durch stärkere Chitinisierung und Verdunkelung erkennbar. Die Verbindung no. I, mit.der Mitte der Gonapophyse, ist hier nicht vorhanden, dafür liegt ihre Endpartie unmittelbar neben einer stark gebogenen Platte, welche das distale Ende der Gonapophyse ist und nur wenig nach aussen vorragt, eine kurze, vorspringende Partie trägt gleichfalls mehrere Börstchen. Der zweite Gelenk (no. 2) liegt hier weiter nach hinten, etwa in der Mitte des äusseren Astes; dieser endet mit dem 3 ten Gelenk, welcher mit einem konischen inneren Fortsatz des gten Segments articuliert, welcher den processus longus repräsentiert wie bei den oben beschriebenen Formen. Am eigentümlichsten sind die Gontapophysen gebildet. Ausser dass ihr Endteil nur sehr wenig vor- 
ragt, zeichnen sie sich dadurch aus, dass an diese sich ein dünner, bandförmiger, wohl im Anfang noch oberflächlich liegender Stab anschliesst, welcher stark gebogen sich nach innen eine lange Strecke fortsetzt und sich mit einer Verdickung zuletzt an die Tragplatte anschmiegt, welche gerade unter dieser Stelle jederseits zahnartig vorspringt. Bei Venturi heisst die Tragplatte apodema del pene, die Gabelplatte sternite genitale, die Gonapophyse bacchetta del pene.

Das Verhalten bei Agr. ambigua ist dem von mobilis sehr ähnlich und ähnliches fand ich auch bei anderen Agromyza-Arten, so albipennis, ferruginosa, niveipennis u.s.w.

Von den übrigen Gattungen ist, was die Genitalien anlangt, nur erst wenig bekannt. Hendel hat vor vielen Jahren schon angegeben, dass der Penis bei verschiedenen Arten von Phytomyza eine verschiedene Form zeigt und SPEIJER hat dies für eine Anzahl bestätigen können. Wegen der Kleinheit der Objekte und wegen der verwickelten Verhältnisse ist es keine leichte Aufgabe die vergleichende Morphologie bei diesen winzigen Fliegen aufzuklären.

\section{LITERATUR}

BrüEr., L. Anatomie und Entwicklungsgeschichte der Geschlechtsausführwege sammt Annexen von Calliphora erythrocephala Zool. Jahrb. Abt. Anat. X. 1897, p. 51 I-618.

Cole, F. R. A Study of the terminal abdominal structures of Male Diptera, Proc. of the California Academy of Sciences, Fourth series, no. 10, 1927.

Feuerborn, H. J. Das Hypopygium „inversum” und „circumversum” der Dipteren. Zool. Anz. LV No. $9 / 10,5$ Dec. '22, p. 189-212.

Hennig, W. Zur Kenntnis der Kopulationsorgane der Tyliden (Micropeziden, Dipt. Acalypt.). Zool. Anz. Bd. ro7, Heft $3 / 4$ 15.7.1934.

Meijere, J. C. H. DE. Verzeichnis der holländischen Agromyzinen. Tịjschr, v. Entom. LXVII, 1924, p. I19-155.

- Die Larven der Agromyzinen. Tijdschr. v. Entom. LXVIII, 1925, p. I95-293 und LXIX, 1926, p. 227-3r8.

- Einige Notizen zu Czerny, Anthomyzidae, Opomyzidae, Tethinidae. Tijdschr. v. Entom. LXXV, 1932, p. 284-288.

Mrall, L. C. and TAylor, T. H. The Structure and Life History of the Holly-fly. Transact. Entom. Soc. of London, 1907, p. 259-283.

Petzold, Wiltrud. Bau und Funktion des Hypopygiums bei den Tachinen, mit bèsonderer Berücksichtigung der Kieferneulentachine (Ernestia rudis Fall.) Inaug. Diss. Jena, I927.

SCHRÄDER, Th. Das Hypopygium „circumversum” von Calliphora erythrocephala. Inaug. Diss. Münster, 1927.

SPEIJER, E. A. M. De hypopygia van eenige Agromyzidae, benevens theoretische beschouwingen over de homologieën van de aanhangsels hiervan, Inaug. Diss. Amsterdam, 1934.

Sturtevant, A. H. The North American Species of Drosophila, Washington IgzI. 